\title{
Análisis de la oferta de ostión japonés en el estado de Baja California Sur (México)
}

Supply Analysis of Japanese Oysters in the State of Baja California Sur (Mexico)

Análise da oferta de ostras japonesas no Estado de Baixa Califórnia Sul (México)

\author{
Mauro Alejandro Monroy Ceseña* \\ Luis Carlos Amador Betancourt ${ }^{\star *}$
}

Fecha de recibido: 18 de febrero de 2015

Fecha de aprobado: 25 de agosto de 2015

Doi: dx.doi.org/10.12804/rev.univ.empresa.30.2016.04

Para citar este artículo: Monroy Ceseña, M. A. \& Amador Betancourt, L. C. (2016). Análisis de la oferta de ostión japonés en el estado de Baja California Sur, México. Universidad \& Empresa, 18(30), 75-96. Doi: dx.doi. org/10.12804/rev.univ.empresa.30.2016.04

\section{RESUMEN}

Esta investigación analiza el comportamiento de la oferta local de ostión japonés en el estado de Baja California Sur y la oferta nacional en México. El estudio es de tipo descriptivo y el diseño, no experimental. Los resultados muestran una tendencia fructífera para los tomadores de decisión en el sector, dentro de un marco estratégico.

Palabras clave: análisis de la oferta, comportamiento de la producción, estimaciones, ostión japonés, valor económico.

Profesor investigador del Departamento Académico de Economía de la Universidad Autónoma de Baja California Sur. Correo electrónico: monroym@uabcs.mx

** Profesor investigador del Departamento Académico de Economía de la Universidad Autónoma de Baja California Sur. Correo electrónico: lamador@uabcs.mx 


\section{ABSTRACT}

This research analyzes the current situation of the supply of Japanese oysters at local level in the State of Baja California Sur and at national level in Mexico. The study is descriptive and the design is non-experimental. The results show a fructiferous trend for decision makers in the sector, within a strategic framework.

Keywords: Supply analysis, production behavior, estimates, Japanese oysters, economic value.

\section{RESUMO}

Esta pesquisa analisa o comportamento existente na oferta de ostras japonesas ao nível local no Estado de Baixa Califórnia Sul e ao nível nacional no México. O estudo realizado é de tipo descritivo e o desenho não experimental. Os resultados determinam uma tendência que é frutífera para os tomadores de decisão no setor, dentro de um marco estratégico. Palavras-chave: análise da oferta, comportamento da produção, estimações, ostra japonesa, valor económico. 


\section{INTRODUCCIÓN}

En la actualidad, los productores pertenecientes a la industria ostrícola en el estado de Baja California Sur (BCS), en México, desarrollan su actividad por medio de procedimientos estrictamente artesanales. Por supuesto, esto dignifica el trabajo realizado, pero, al mismo tiempo, limita las unidades producidas y restringe las posibles técnicas de comercialización que podrían utilizarse en el mercado meta, con el fin de ampliar el horizonte comercial.

La industria ostrícola ha sido un partícipe importante de la economía sudcaliforniana, debido, entre otras cosas, a las condiciones geográficas con las que cuenta BCS que hacen factible el desarrollo de ese proceso productivo, en particular, en las zonas colindantes a las costas del territorio estatal.

La producción de ostión reportada por la Secretaría de agricultura, ganadería, desarrollo rural, pesca y alimentación (Sagarpa) en los últimos años, en las oficinas situadas en los municipios del estado de BCS (La Paz, Los Cabos, Comondú, Loreto y Mulegé), presentan un índice bastante aceptable. De hecho, al estimar su proyección unitaria, muestra una pendiente positiva.

El desarrollo de trabajo de investigación procura responder al obje- tivo de identificar cuál es la oferta de ostión japonés producida en el estado de BCS y, a su vez, hacer un comparativo entre las oficinas. Con este fin, el presente documento se ha estructurado en tres secciones básicas. En la primera se presenta la metodología del trabajo; en la segunda, los resultados del estudio llevado a cabo y, en la tercera, las principales conclusiones derivadas.

\section{METODOLOGÍA}

Esta investigación es de tipo descriptivo y su diseño fue no experimental (Hernández Sampieri, Fernández Collado \& Baptista Lucio, 2004). Para la recolección de los datos, fue necesario solicitar información oficial a las instancias correspondientes, en particular, la Sagarpa, la Comisión Nacional de Acuacultura y Pesca (Conapesca), el Comité de Sanidad Acuícola de Baja California Sur y el Sistema Producto Ostión de Baja California Sur. Este material recibió un tratamiento estadístico para hacer las interpretaciones correspondientes.

\section{RESULTADOS: ANÁLISIS DE LA OFERTA DE OSTIÓN JAPO- NÉS EN EL ESTADO DE BCS}

El ostión japonés (Crassostrea gigas) es una de las especies de molusco bivalvo más apreciada en todo el mundo. Esto se debe a su excelente sabor, a la rapidez de su crecimiento 
y a su gran adaptabilidad. En virtud de estas cualidades, el ostión japonés se ha introducido con éxito como una especie repobladora en muy diversas regiones, entre las que es posible destacar la costa pacífica de Estados Unidos, la provincia de Columbia Británica en Canadá, Nueva Zelanda, Reino Unido, etc. (Bourne, 1979; Chew, 1979; Mann, 1979; Stickney, 1979).

En México, el ostión japonés fue introducido en las costas de Baja California con resultados satisfactorios (Islas Olivares, 1975; 1982). El crecimiento de la producción de este molusco en el país, entre 2006 y 2011, puede observarse en la tabla 1 .

Tabla 1. Oferta nacional de ostión, período 2006-2011

\begin{tabular}{|c|c|c|c|}
\hline \multicolumn{2}{|c|}{ Producción México } & $\%$ & \multirow{2}{*}{ Media } \\
\hline 2006 & 46,762 & $17 \%$ & \\
\hline 2007 & 50,264 & $18 \%$ & \\
\hline 2008 & 44,452 & $16 \%$ & \multirow{2}{*}{$46,882.33$} \\
\hline 2009 & 42,250 & $15 \%$ & \\
\hline 2010 & 50,715 & $18 \%$ & \\
\hline 2011 & 46,851 & $17 \%$ & \\
\hline
\end{tabular}

Fuente: elaboración propia con base en datos del PortalInformativoAcuícola(Acuasesor-Conapesca).

La tabla 1 muestra una repartición casi equitativa de los índices de producción anual mexicana durante el mencionado período. $\mathrm{Al}$ obtener un dato promedio es posible visualizar que, durante un $66 \%$ del tiempo, la producción de estos moluscos bivalvos estuvo por debajo de la media.

El comportamiento de la producción de ostiones en territorio mexicano ha presentado subidas y bajadas, lo que puede interpretarse estadísticamente por medio de indicadores de cambio. Este es el caso que se aprecia en la tabla 2. En ella se observan los comportamientos porcentuales de la producción de ostión en el período 2006-2011. En los años 2008, 2009 y 2011 hubo decrementos en relación con la producción de años anteriores.

Tabla 2. Cambio porcentual en la producción nacional de ostión, período 2006-2011

\begin{tabular}{|c|c|c|}
\hline \multicolumn{2}{|c|}{ Producción México } & Cambio \% \\
\hline 2006 & 46,762 & \\
\hline 2007 & 50,264 & $7 \%$ \\
\hline 2008 & 44,452 & $-12 \%$ \\
\hline 2009 & 42,250 & $-5 \%$ \\
\hline 2010 & 50,715 & $20 \%$ \\
\hline 2011 & 46,851 & $-8 \%$ \\
\hline
\end{tabular}

Fuente: elaboración propia con base en datos del Portal Informativo Acuícola (AcuasesorConapesca).

En el escenario estatal, en los últimos años, la producción del estado de BCS ha obtenido los resultados que se incorporan en la tabla 3. 
Tabla 3. Producción de ostión en BCS, período 2006-2011

\begin{tabular}{|c|c|c|c|}
\hline & Producción BCS & $\%$ & Media \\
\hline 2006 & 335.76 & $13 \%$ & \multirow{6}{*}{441.91} \\
\hline 2007 & 473.28 & $18 \%$ & \\
\hline 2008 & 321.46 & $12 \%$ & \\
\hline 2009 & 404.32 & $15 \%$ & \\
\hline 2010 & 665.31 & $25 \%$ & \\
\hline 2011 & 451.32 & $17 \%$ & \\
\hline
\end{tabular}

Fuente: elaboración propia con base en datos del Portal Informativo Acuícola (AcuasesorConapesca).

Al promediar los descensos nacionales, ocurridos en los años 2008, 2009 y 2011, la disminución es de $8,33 \%$ (tabla 2). Entre 2010 y 2011 , en el estado, hubo un descenso en la oferta de ostión japonés de $8 \%$. Esto puede interpretarse como una situación de alarma para los tomadores de decisión en el ámbito pesquero de México (Conapesca).

En cuanto al ámbito estatal —objeto de interés de este trabajo de investigación-, la oferta del ostión puede identificarse por medio del acumulativo registrado en las oficinas en las que se reporta su producción mensual y anual. A continuación, se muestran los principales resultados estatales, en particular, para el ejercicio de 2013, de acuerdo con las oficinas de las ciudades de Cabo San Lucas, Ciudad Constitución, Guerrero Negro, La Paz, el cabo Punta Abreojos y la ciudad Santa Rosalía.

\subsection{Oficina en Cabo San Lucas (CSL)}

La producción de ostión en la oficina de CSL para el año 2013 se aprecia en la tabla 4:

Tabla 4. Producción de ostión, oficina de CSL, año 2013

\begin{tabular}{|l|c|}
\hline Enero & 3,700 \\
\hline Febrero & 5,330 \\
\hline Marzo & 4,300 \\
\hline Mayo & 1,150 \\
\hline Septiembre & 1,345 \\
\hline Octubre & 1,832 \\
\hline Noviembre & 3,970 \\
\hline Diciembre & 3,000 \\
\hline
\end{tabular}

Fuente: elaboración propia con base en datos de la Subdelegación de Pesca, BCS.

Los resultados de la oficina de CSL, en el estado de BCS, muestran que, aunque se encuentran por encima de la media generada para el año 2013, más de $60 \%$ de los meses evidencian un resultado que no puede ser considerado como positivo (figura 1). 
Figura 1. Producción de ostión registrada en la oficina de CSL, año 2013

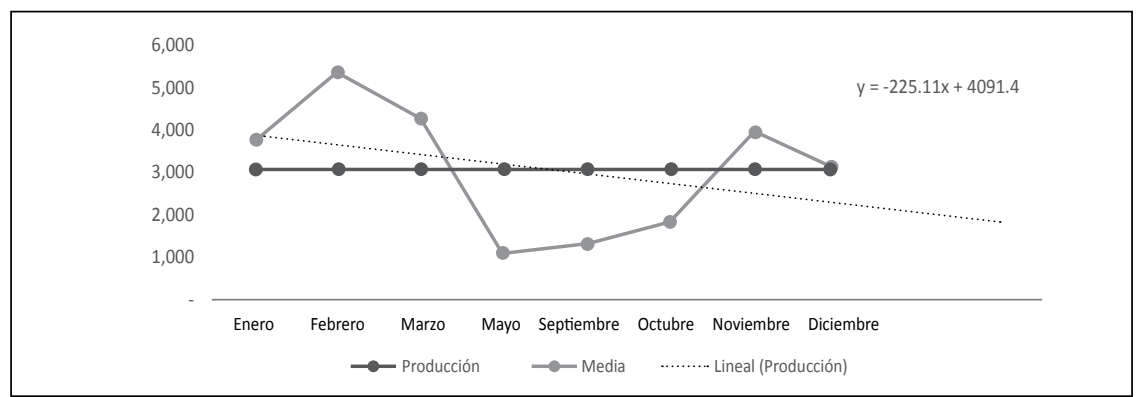

Fuente: elaboración propia.

Los resultados del comportamiento gularidad en la producción registradel cambio porcentual, considera- dos en la oficina de CSL (tabla 5 y do mes a mes, no ocultan la irre- figura 2).

Tabla 5. Comportamiento porcentual de la producción de ostión registrada en la oficina de CSL, año 2013

\begin{tabular}{|l|c|c|}
\hline Enero & 3,700 & \\
\hline Febrero & 5,330 & $44.05 \%$ \\
\hline Marzo & 4,300 & $-19.32 \%$ \\
\hline Mayo & 1,150 & $-73.26 \%$ \\
\hline Septiembre & 1,345 & $16.96 \%$ \\
\hline Octubre & 1,832 & $36.21 \%$ \\
\hline Noviembre & 3,970 & $116.70 \%$ \\
\hline Diciembre & 3,000 & $-24.43 \%$ \\
\hline
\end{tabular}

Fuente: elaboración propia con base en datos de la Subdelegación de Pesca, BCS.

Figura 2. Comportamiento de cambio porcentual en la producción en la oficina de CSL, año 2013

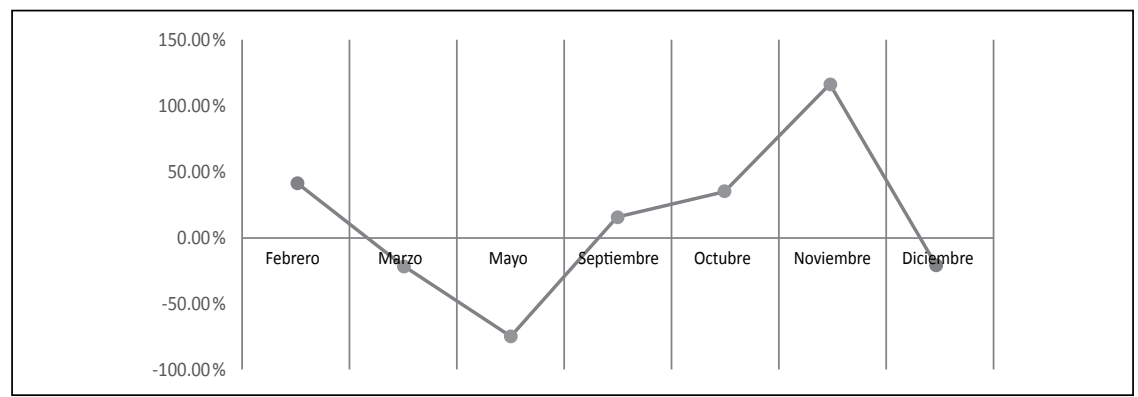

Fuente: elaboración propia. 
Por otra parte, en cuanto al valor económico, el comportamiento de la producción de ostión durante el año 2013 tuvo el comportamiento que se incorpora en la tabla 6 y la figura 3.

Tabla 6. Comportamiento del valor económico de la producción de ostión en la oficina de CSL, año 2013

\begin{tabular}{|l|r|}
\hline Enero & $\$ 29,600.00$ \\
\hline Febrero & $\$ 29,140.00$ \\
\hline Marzo & $\$ 34,400.00$ \\
\hline Mayo & $\$ 9,200.00$ \\
\hline Septiembre & $\$ 10,760.00$ \\
\hline Octubre & $\$ 14,656.00$ \\
\hline Noviembre & $\$ 31,760.00$ \\
\hline Diciembre & $\$ 24,000.00$ \\
\hline
\end{tabular}

Fuente: elaboración propia con base en datos de la Subdelegación de Pesca, BCS.

Figura 3. Valor económico registrado en la oficina de CSL, año 2013

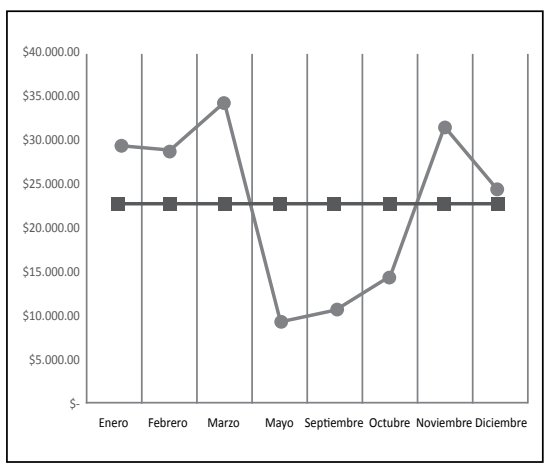

Fuente: elaboración propia con base en datos de la Subdelegación de Pesca, BCS.

Como se observa en la tabla 6 , en la oficina de CSL-BCS, se reportó un total $\$ 183.516$ en 2013. Este se vio reflejado, en su mayoría (81\%), en los meses de enero a marzo, noviembre y diciembre.

\subsection{Oficina en Ciudad Constitución}

La producción de ostión en la oficina de Ciudad Constitución para el año 2013 se muestra en la tabla 7.

Tabla 7. Producción de ostión, oficina de Ciudad Constitución, año 2013

\begin{tabular}{|l|r|}
\hline Enero & 3,000 \\
\hline Febrero & 5,000 \\
\hline Marzo & 25,000 \\
\hline Abril & 10,000 \\
\hline Mayo & 10,000 \\
\hline Junio & 15,000 \\
\hline Julio & 5,000 \\
\hline Agosto & 2,100 \\
\hline Noviembre & 11,000 \\
\hline Diciembre & 5,000 \\
\hline
\end{tabular}

Fuente: elaboración propia con base en datos de la Subdelegación de Pesca, BCS.

Los resultados que se aprecian en el registro de la oficina de Ciudad Constitución señalan que un $50 \%$ de los meses de 2013 en los que se registró producción de ostión reflejan un comportamiento irregular. Una estimación mensual permite identificar la presencia de un comportamiento negativo (figura 4): 
Figura 4. Producción registrada en la oficina de Ciudad Constitución, año 2013

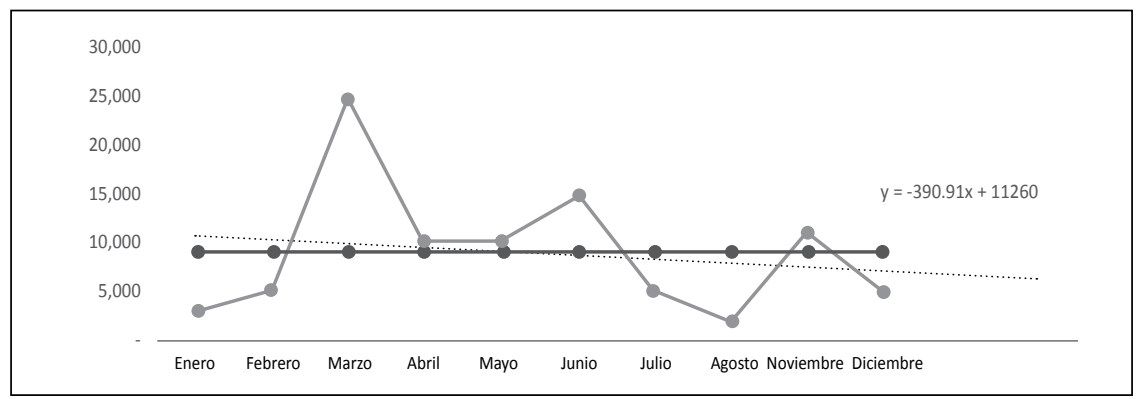

Fuente: elaboración propia.

Figura 5. Comportamiento de cambio porcentual en la producción en la oficina de Ciudad Constitución, año 2013

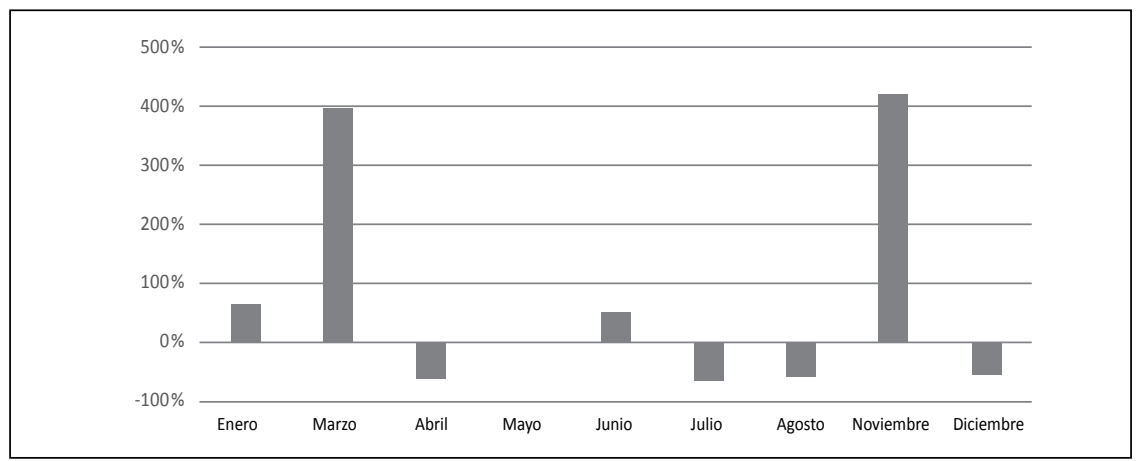

Fuente: elaboración propia con base en datos de la Subdelegación de Pesca, BCS.

Los resultados en materia del comportamiento de cambio porcentual por mes indican la irregularidad de la producción. Estos se plasman en la tabla 8 y en la figura 5 .

Por otra parte, en lo relacionado con el valor económico, el comportamiento de la producción de ostión durante 2013, en la oficina de Ciudad Constitución, tuvo el comportamiento señalado en la tabla 9.
Tabla 8. Comportamiento de cambio porcentual en la producción registrada en la oficina de Ciudad Constitución, año 2013

\begin{tabular}{|l|c|c|}
\hline Enero & 3,000 & \\
\hline Febrero & 5,000 & $67 \%$ \\
\hline Marzo & 25,000 & $400 \%$ \\
\hline Abril & 10,000 & $-60 \%$ \\
\hline Mayo & 10,000 & $0 \%$ \\
\hline Junio & 15,000 & $50 \%$ \\
\hline
\end{tabular}




\begin{tabular}{|l|c|c|}
\hline Julio & 5,000 & $-67 \%$ \\
\hline Agosto & 2,100 & $-58 \%$ \\
\hline Noviembre & 11,000 & $424 \%$ \\
\hline Diciembre & 5,000 & $-55 \%$ \\
\hline
\end{tabular}

Fuente: elaboración propia con base en datos de la Subdelegación de Pesca, BCS.

Tabla 9. Comportamiento del valor económico de la producción de ostión en la oficina de Ciudad Constitución, año 2013

\begin{tabular}{|l|r|}
\hline Enero & $\$ 29,600.00$ \\
\hline Febrero & $\$ 29,140.00$ \\
\hline Marzo & $\$ 34,400.00$ \\
\hline Mayo & $\$ 9,200.00$ \\
\hline Septiembre & $\$ 10,760.00$ \\
\hline Octubre & $\$ 14,656.00$ \\
\hline Noviembre & $\$ 31,760.00$ \\
\hline Diciembre & $\$ 24,000.00$ \\
\hline
\end{tabular}

Fuente: elaboración propia con base en datos de la Subdelegación de Pesca, BCS.

\subsection{Oficina de Guerrero Negro}

La producción de ostión en la oficina de Guerrero Negro para el año 2013 se indica en la tabla 10 :

Tabla 10. Producción de ostión en la oficina de Guerrero Negro, año 2013

\begin{tabular}{|l|r|}
\hline Marzo & 900 \\
\hline Mayo & 400 \\
\hline Julio & 2.100 \\
\hline Agosto & 750 \\
\hline Septiembre & 900 \\
\hline Diciembre & 300 \\
\hline
\end{tabular}

Fuente: elaboración propia con base en datos de la Subdelegación de Pesca, BCS.

Los resultados de la oficina de Guerrero Negro muestran que más de $85 \%$ de los meses de 2013 en los que se registró producción de ostión están por debajo de la media. En el

Figura 6. Producción registrada en la oficina de Guerrero Negro

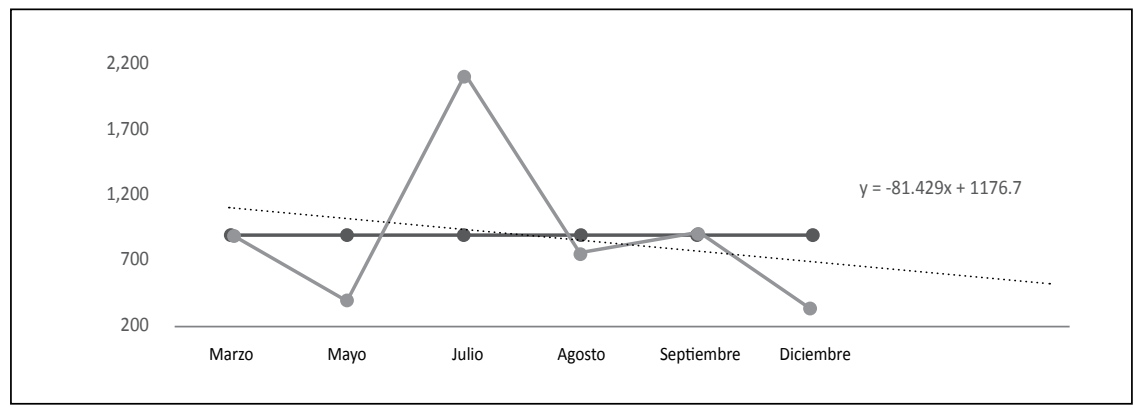

Fuente: elaboración propia.

momento de establecer una estima- de un comportamiento negativo (fición mensual, el resultado da cuenta gura 6). 
Los resultados del comportamiento tran irregularidad en la producción de cambio porcentual por mes mues- registrada (tabla 11 y figura 7).

Tabla 11. Comportamiento de cambio porcentual en la producción registrada en la oficina de Guerrero Negro, año 2013

\begin{tabular}{|l|c|c|}
\hline Marzo & 900 & \\
\hline Mayo & 400 & $-55.56 \%$ \\
\hline Julio & 2.100 & $425.00 \%$ \\
\hline Agosto & 750 & $-64.29 \%$ \\
\hline Septiembre & 900 & $20.00 \%$ \\
\hline Diciembre & 300 & $-66.67 \%$ \\
\hline
\end{tabular}

Fuente: elaboración propia con base en datos de la Subdelegación de Pesca, BCS.

Figura 7. Comportamiento de cambio porcentual en la producción en la oficina de Guerrero Negro, año 2013

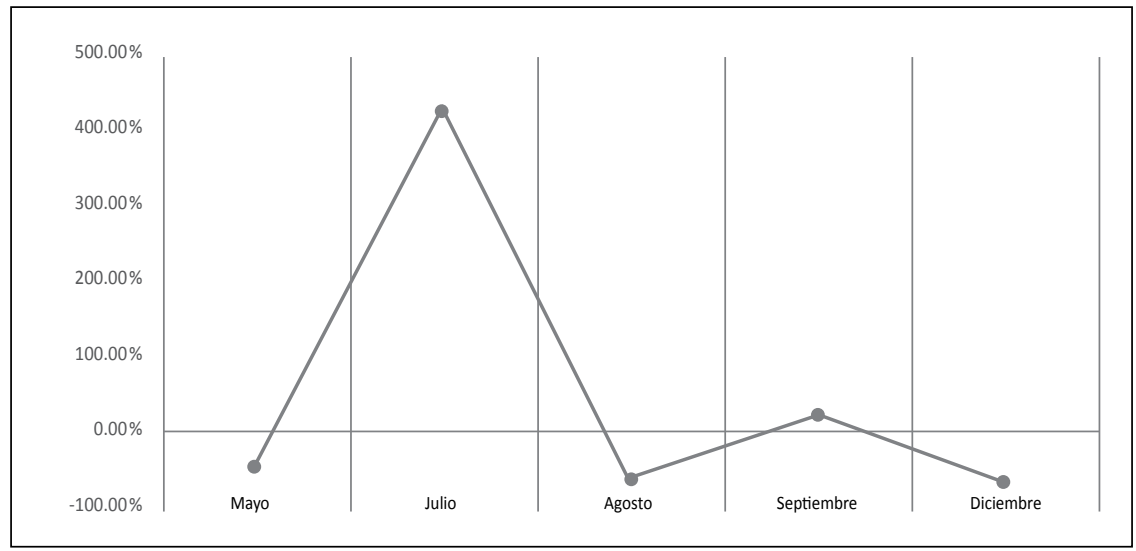

Fuente: elaboración propia con base en datos de la Subdelegación de Pesca, BCS.

En lo relacionado con el valor económico, el comportamiento de la producción de ostión en 2013, en la oficina de Guerrero Negro, se presentó de la siguiente manera (tabla 12 y figura 8$)$.
Tabla 12. Comportamiento del valor económico de la producción de ostión en la oficina de Guerrero Negro, año 2013

\begin{tabular}{|l|r|}
\hline Marzo & $\$ 6,300.00$ \\
\hline Mayo & $\$ 2,800.00$ \\
\hline Julio & $\$ 14,700.00$ \\
\hline
\end{tabular}




\begin{tabular}{|l|r|}
\hline Agosto & $\$ 5,250.00$ \\
\hline Septiembre & $\$ 6,300.00$ \\
\hline Diciembre & $\$ 2,100.00$ \\
\hline
\end{tabular}

Fuente: elaboración propia con base en datos de la Subdelegación de Pesca, BCS.

Como se observa en la tabla 12, en 2013, la oficina de Guerrero Negro reportó un total de $\$ 37.450$. Un $70 \%$ de este valor se configuró en los meses de julio a septiembre.

Figura 8. Valor económico registrado en la oficina de Guerrero Negro, año 2013

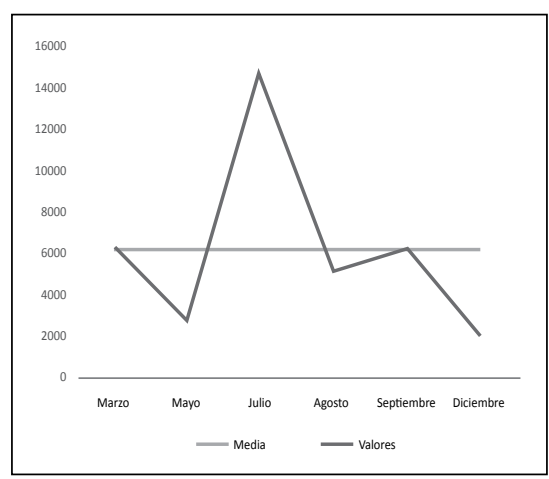

Fuente: elaboración propia con base en datos de la Subdelegación de Pesca, BCS.

\subsection{Oficina de La Paz}

La producción de ostión en la oficina de La Paz se sintetiza en la tabla 13.

Tabla 13. Producción de ostión en la oficina de La Paz, año 2013

\begin{tabular}{|l|r|}
\hline Marzo & 530 \\
\hline Junio & 500 \\
\hline Julio & 1,610 \\
\hline Agosto & 12,855 \\
\hline Septiembre & 7,000 \\
\hline Octubre & 5,000 \\
\hline Noviembre & 6,615 \\
\hline Diciembre & 500 \\
\hline
\end{tabular}

Fuente: elaboración propia con base en datos de la Subdelegación de Pesca, BCS.

Los resultados en la oficina de $\mathrm{La}$ Paz muestran que, gracias a que durante un $85 \%$ de los meses de 2013 se registró producción de ostión por encima de la media generada, es posible identificar la existencia de un resultado positivo (figura 9).

Figura 9. Producción registrada en la oficina de La Paz, año 2013

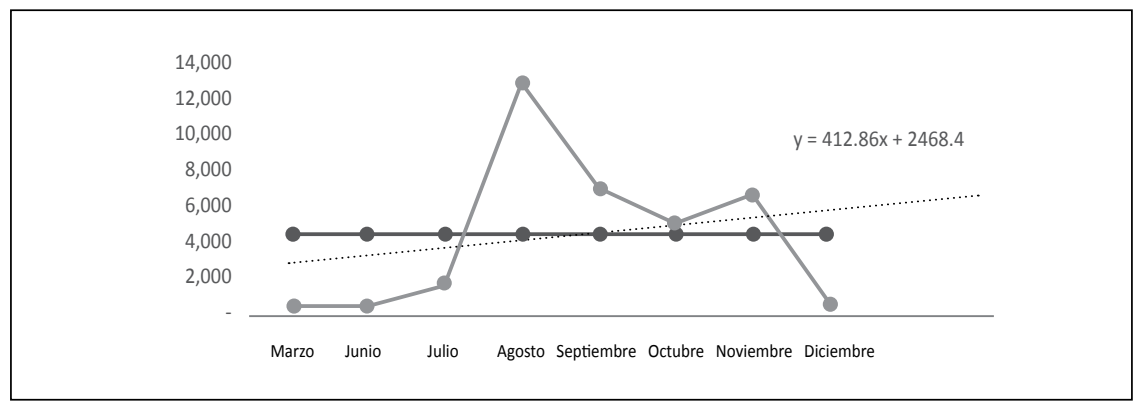

Fuente: elaboración propia. 
Los resultados del comportamiento de cambio porcentual por mes muestran la forma en la que la producción de ostión registrada en la oficina de
La Paz da cuenta de una mayor regularidad que en las anteriores oficinas consideradas. Esto se plasma en la figura 9 y en la tabla 14 .

Tabla 14. Comportamiento de cambio porcentual en la producción registrada en la oficina de La Paz, año 2013

\begin{tabular}{|l|c|c|}
\hline Marzo & 530 & \\
\hline Junio & 500 & $-5.66 \%$ \\
\hline Julio & 1,610 & $222.00 \%$ \\
\hline Agosto & 12,855 & $698.45 \%$ \\
\hline Septiembre & 7,000 & $-45.55 \%$ \\
\hline Octubre & 5,000 & $-28.57 \%$ \\
\hline Noviembre & 6,615 & $32.30 \%$ \\
\hline Diciembre & 500 & $-92.44 \%$ \\
\hline
\end{tabular}

Fuente: elaboración propia con base en datos de la Subdelegación de Pesca, BCS.

Figura 10. Comportamiento de cambio porcentual en la producción en la oficina de La Paz, año 2013

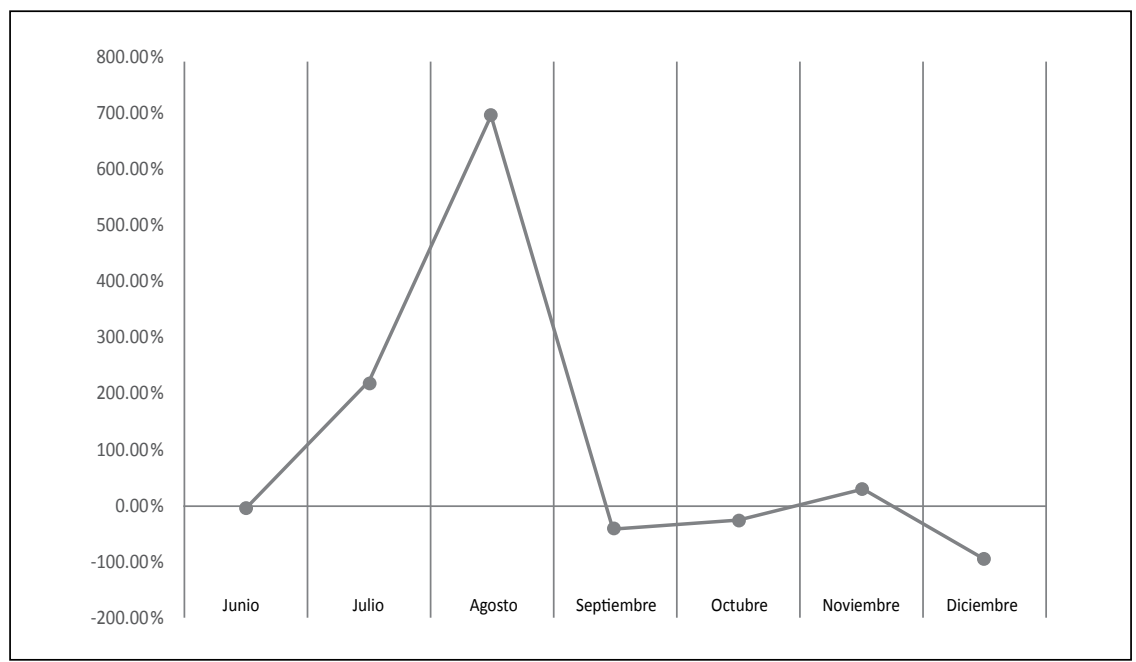

Fuente: elaboración propia con base en datos de la Subdelegación de Pesca, BCS.

El comportamiento económico de la oficina de La Paz se muestra en la la producción de ostión en 2013 en tabla 15 y en la figura 11. 
Tabla 15. Comportamiento del valor económico de la producción de ostión en la oficina de La Paz, año 2013

\begin{tabular}{|l|r|}
\hline Marzo & $\$ 3,710.00$ \\
\hline Junio & $\$ 4,000.00$ \\
\hline Julio & $\$ 12,880.00$ \\
\hline Agosto & $\$ 120,440.00$ \\
\hline Septiembre & $\$ 70,000.00$ \\
\hline Octubre & $\$ 50,000.00$ \\
\hline Noviembre & $64,920.00$ \\
\hline Diciembre & $4,000.00$ \\
\hline
\end{tabular}

Fuente: elaboración propia con base en datos de la Subdelegación de Pesca, BCS.

\section{Figura 11. Valor económico registrado} en la oficina de La Paz

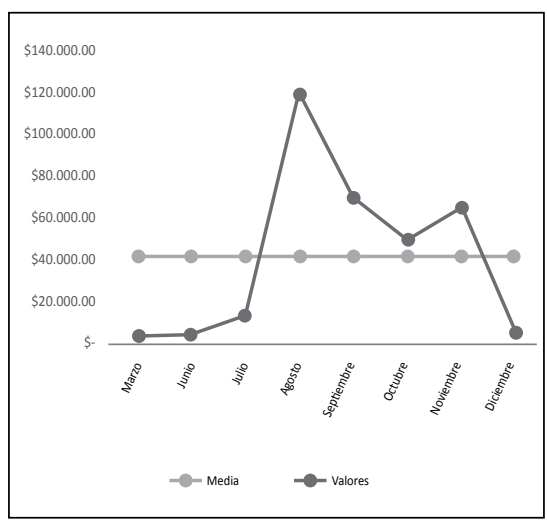

Fuente: elaboración propia con base en datos de la Subdelegación de Pesca, BCS.

Durante todo el año, en la oficina de La Paz se reportaron $\$ 329.950$. Un $93 \%$ se obtuvo en los meses de agosto a noviembre (tabla 15 ).

\subsection{Oficina de Punta Abreojos}

La producción de ostión en la oficina de Punta Abreojos, para el año 2013, se muestra en la tabla 16.

Tabla 16. Producción de ostión, oficina de Punta Abreojos, año 2013

\begin{tabular}{|l|r|}
\hline Enero & 1,650 \\
\hline Febrero & 2,596 \\
\hline Marzo & 4,050 \\
\hline Abril & 7,400 \\
\hline Mayo & 15,560 \\
\hline Junio & 3,915 \\
\hline Julio & 3,795 \\
\hline Agosto & 5,440 \\
\hline Septiembre & 4,350 \\
\hline Octubre & 2,500 \\
\hline Noviembre & 2,000 \\
\hline Diciembre & 980 \\
\hline
\end{tabular}

Fuente: elaboración propia con base en datos de la Subdelegación de Pesca, BCS.

Los resultados de la oficina de Punta Abreojos indican que durante un $75 \%$ de los meses considerados en 2013 se registró una producción de ostión por debajo de la media. Esto fue suficiente para establecer una estimación mensual con resultado negativo (figura 12). 
Figura 12. Producción registrada en la oficina de Punta Abreojos, año 2013

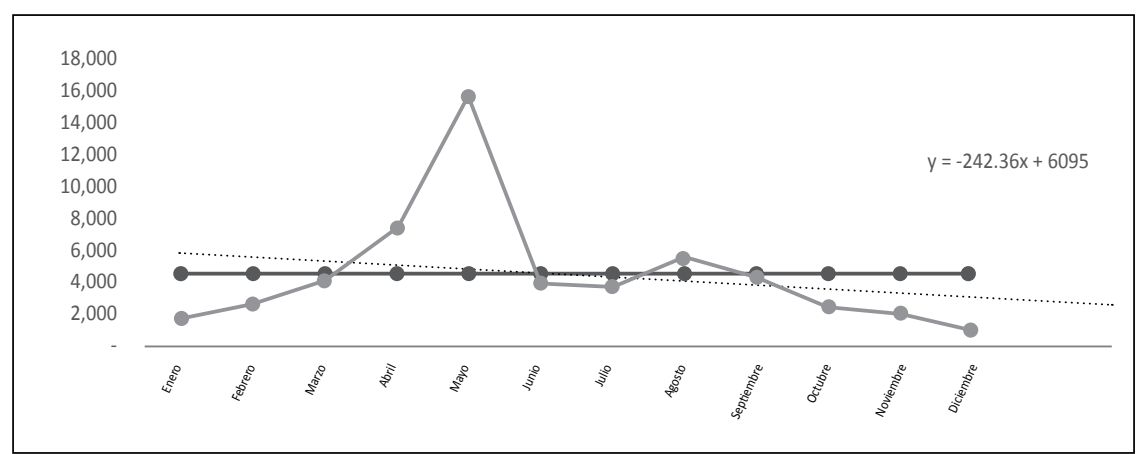

Fuente: elaboración propia.

Los resultados del comportamiento en la oficina de La Paz tiene una de cambio porcentual por mes se- mayor regularidad. Esto se plasma ñalan que la producción registrada en la tabla 17 y en la figura 13.

Tabla 17. Comportamiento de cambio porcentual en la producción registrada en la oficina de Punta Abreojos, año 2013

\begin{tabular}{|l|c|c|}
\hline Enero & 1,650 & $57.33 \%$ \\
\hline Febrero & 2,596 & $56.01 \%$ \\
\hline Marzo & 4,050 & $82.72 \%$ \\
\hline Abril & 7,400 & $110.27 \%$ \\
\hline Mayo & 15,560 & $-74.84 \%$ \\
\hline Junio & 3,915 & $-3.07 \%$ \\
\hline Julio & 3,795 & $43.35 \%$ \\
\hline Agosto & 5,440 & $-20.04 \%$ \\
\hline Septiembre & 4,350 & $-42.53 \%$ \\
\hline Octubre & 2,500 & $-20.00 \%$ \\
\hline Noviembre & 2,000 & $-51.00 \%$ \\
\hline Diciembre & 980 & $-P e 0$ \\
\hline
\end{tabular}

Fuente: elaboración propia con base en datos de la Subdelegación de Pesca, BCS. 
Figura 13. Comportamiento de cambio porcentual en la producción en la oficina de Punta Abreojos, año 2013

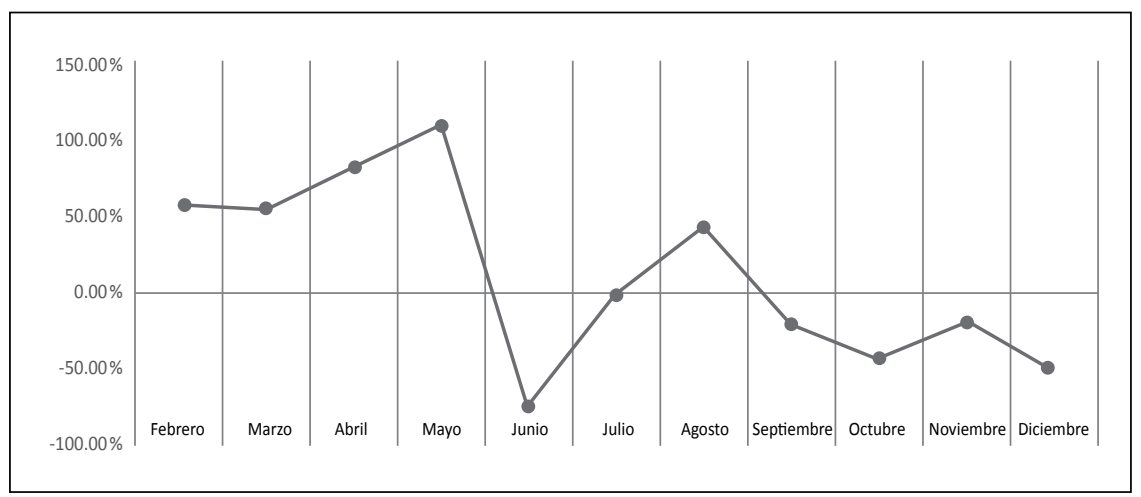

Fuente: elaboración propia con base en datos de la Subdelegación de Pesca, BCS.

El comportamiento económico de la oficina de Punta Abreojos se presenproducción de ostión en 2013, en la ta en la tabla 18 y en la figura 14.

Tabla 18. Comportamiento del valor económico de la producción de ostión en la oficina de Punta Abreojos, año 2013

\begin{tabular}{|l|r|}
\hline Enero & $\$ 61,050.00$ \\
\hline Febrero & $\$ 96,052.00$ \\
\hline Marzo & $\$ 149,850.00$ \\
\hline Abril & $\$ 273,800.00$ \\
\hline Mayo & $\$ 281,580.00$ \\
\hline Junio & $\$ 144,855.00$ \\
\hline Julio & $\$ 140,415.00$ \\
\hline Agosto & $\$ 164,100.00$ \\
\hline Septiembre & $\$ 160,950.00$ \\
\hline Octubre & $\$ 94,300.00$ \\
\hline Noviembre & $\$ 74,000.00$ \\
\hline Diciembre & $\$ 36,260.00$ \\
\hline
\end{tabular}

Fuente: elaboración propia con base en datos de la Subdelegación de Pesca, BCS. 
Figura 14. Valor económico registrado en la oficina de Punta Abreojos, año 2013

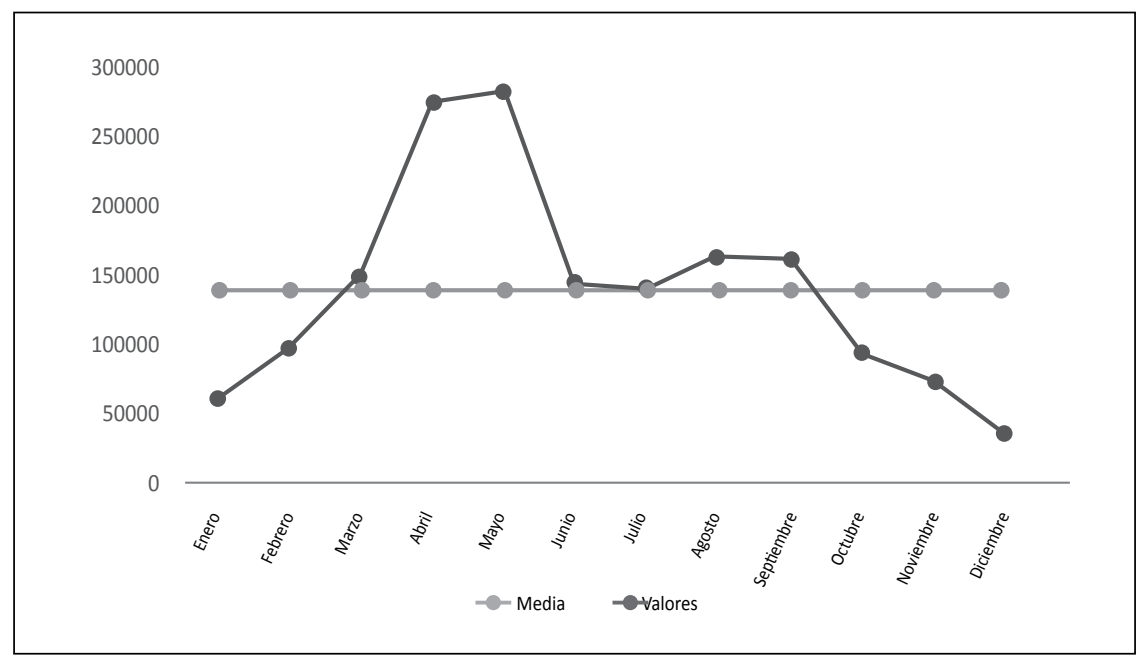

Fuente: elaboración propia con base en datos de la Subdelegación de Pesca, BCS.

En 2013, en la oficina de Punta Abreojos, se reportaron \$1.677.212 (tabla 18). Un 70\% de este total se alcanzó entre los meses de abril y septiembre.

\subsection{Oficina de Santa Rosalía}

La producción de ostión en la oficina de Santa Rosalía para el año 2013 se muestra en la tabla 19.

Tabla 19. Producción de ostión, oficina de Santa Rosalía, año 2013

\begin{tabular}{|l|c|}
\hline Marzo & 15,100 \\
\hline Abril & 18,130 \\
\hline Mayo & 1,000 \\
\hline Julio & 4,000 \\
\hline Agosto & 4,000 \\
\hline Septiembre & 4,000 \\
\hline Octubre & 24,305 \\
\hline Noviembre & 16,749 \\
\hline Diciembre & 11,717 \\
\hline
\end{tabular}

Fuente: elaboración propia con base en datos de la Subdelegación de Pesca, BCS. 
Los resultados en la oficina de Santa Rosalía señalan que un $85 \%$ de los meses estuvo por encima de la me- dia. Esto permitió establecer una estimación mensual con un resultado positivo (figura 15).

Figura 15. Producción registrada en la oficina de Santa Rosalía, año 2013

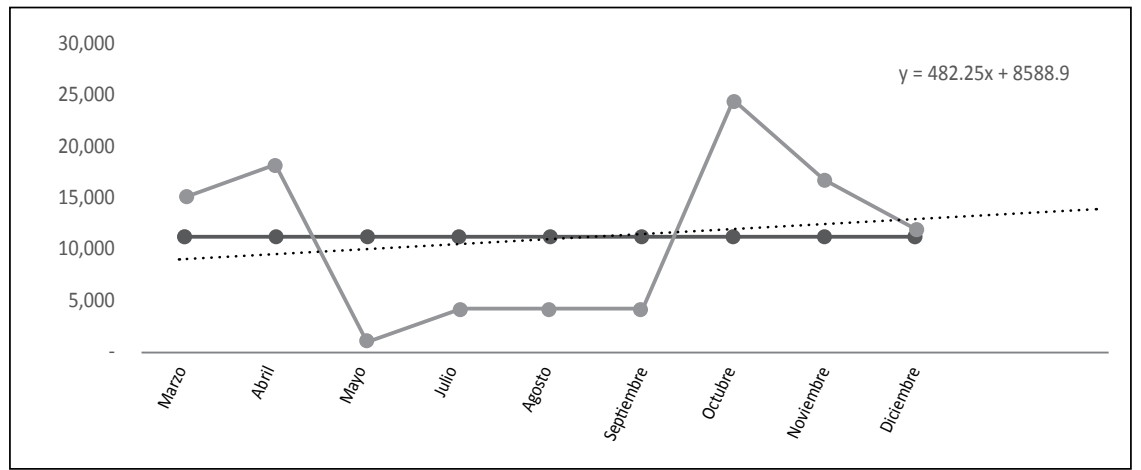

Fuente: elaboración propia con base en datos de la Subdelegación de Pesca, BCS.

Los resultados del comportamiento la oficina de Santa Rosalía tiene una de cambio porcentual por mes indi- mayor regularidad. Estos se aprecan que la producción registrada en cian en la tabla 20 y en la figura 16.

Tabla 20. Comportamiento de cambio porcentual en la producción registrada en la oficina de Santa Rosalía, año 2013

\begin{tabular}{|l|c|c|}
\hline Marzo & 15,100 & \\
\hline Abril & 18,130 & $20.07 \%$ \\
\hline Mayo & 1,000 & $-94.48 \%$ \\
\hline Julio & 4,000 & $300.00 \%$ \\
\hline Agosto & 4,000 & $0.00 \%$ \\
\hline Septiembre & 4,000 & $0.00 \%$ \\
\hline Octubre & 24,305 & $507.63 \%$ \\
\hline Noviembre & 16,749 & $-31.09 \%$ \\
\hline Diciembre & 11,717 & $-30.04 \%$ \\
\hline
\end{tabular}

Fuente: elaboración propia con base en datos de la Subdelegación de Pesca, BCS. 
Figura 16. Comportamiento de cambio porcentual en la producción en la oficina de Santa Rosalía, año 2013

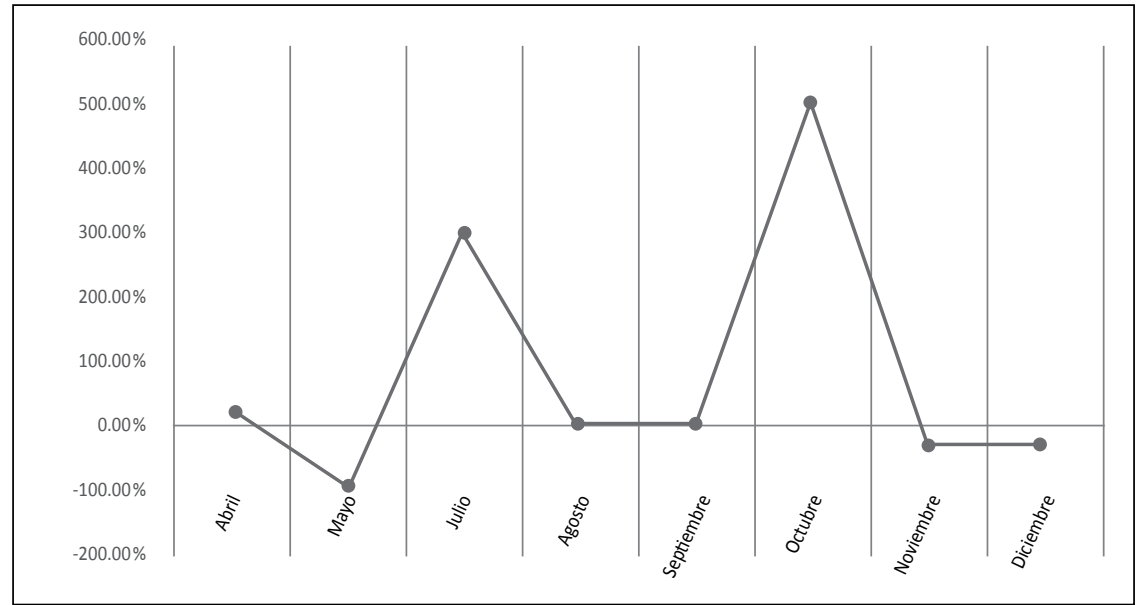

Fuente: elaboración propia con base en datos de la Subdelegación de Pesca, BCS.

El comportamiento económico de la oficina de Santa Rosalía, se sintela producción de ostión en 2013, en tiza en la tabla 21 y en la figura 17.

Tabla 21. Producción de ostión en la oficina de Santa Rosalía, año 2013

\begin{tabular}{|l|r|}
\hline Marzo & $\$ 246,500.00$ \\
\hline Abril & $\$ 271.950 .00$ \\
\hline Mayo & $\$ 20,000.00$ \\
\hline Julio & $\$ 80,000.00$ \\
\hline Agosto & $\$ 80,000.00$ \\
\hline Septiembre & $\$ 80,000.00$ \\
\hline Octubre & $\$ 585,125.00$ \\
\hline Noviembre & $\$ 414,725.00$ \\
\hline Diciembre & $\$ 272,925.00$ \\
\hline
\end{tabular}

Fuente: elaboración propia con base en datos de la Subdelegación de Pesca, BCS. 
Figura 17. Valor económico registrado en la oficina de Santa Rosalía, año 2013

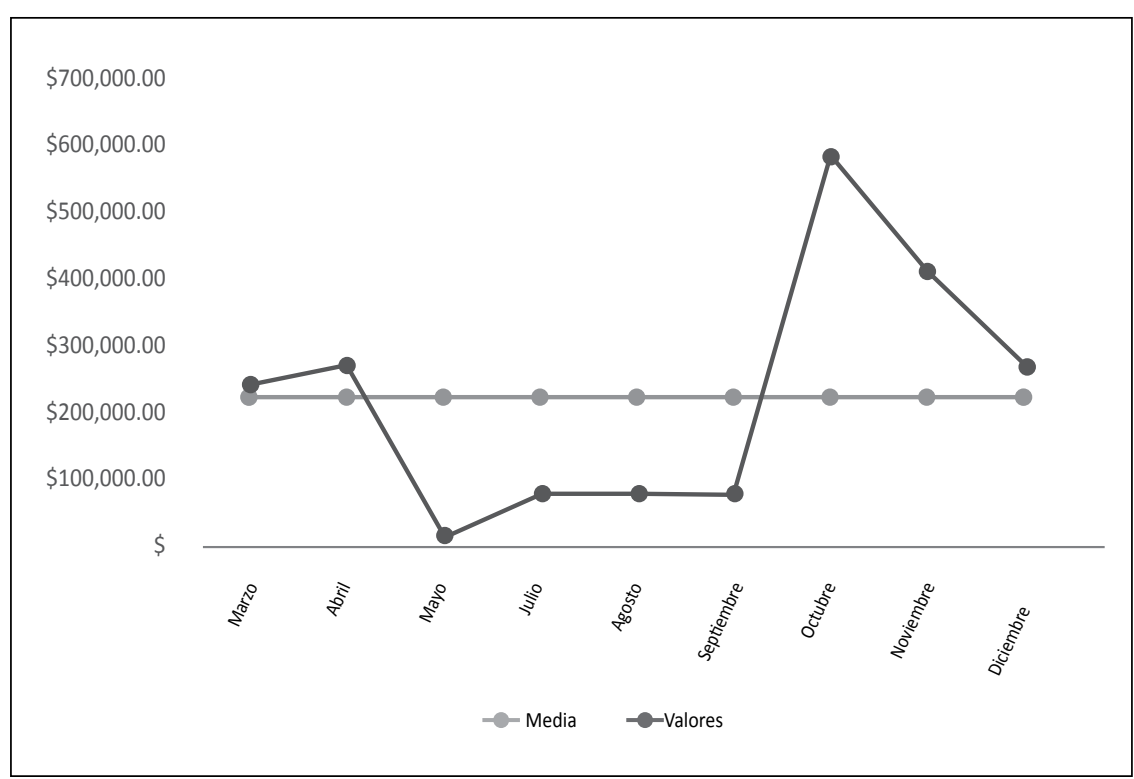

Fuente: elaboración propia con base en datos de la Subdelegación de Pesca, BCS.

Tabla 22. Producción histórica generada en el período 2009-2013 en las oficinas del estado de BCS

\begin{tabular}{|c|c|c|c|c|c|c|c|c|}
\hline & $\begin{array}{c}\text { Bahía } \\
\text { Tortugas }\end{array}$ & $\begin{array}{c}\text { Cabo San } \\
\text { Lucas }\end{array}$ & $\begin{array}{c}\text { Ciudad } \\
\text { Constitución }\end{array}$ & $\begin{array}{c}\text { Guerrero } \\
\text { Negro }\end{array}$ & La Paz & $\begin{array}{c}\text { Punta } \\
\text { Abejorros }\end{array}$ & $\begin{array}{c}\text { Santa } \\
\text { Rosalía }\end{array}$ & \\
\hline 2009 & 7,732 & 82,498 & 56,000 & 3,450 & 213,895 & 71,802 & 221,560 & \\
\hline 2010 & 542 & 40,349 & 58,655 & NR & 85,280 & 110,443 & 490,523 & \\
\hline 2011 & 5,620 & 65,872 & 77,000 & 10,310 & 49,157 & 86,696 & 255,425 & \\
\hline 2012 & 6,500 & 22,469 & 55,000 & 17,130 & 74,880 & 84,690 & 498,721 & \\
\hline 2013 & 4,405 & 24,627 & 91,100 & 5,350 & 34,880 & 54,541 & 99,001 & \\
\hline TOTAL & 24,799 & 235,815 & 337,755 & 36,240 & 458,092 & 408,172 & $1,565,230$ & $3,066,103$ \\
\hline$\%$ & $1 \%$ & $8 \%$ & $11 \%$ & $1 \%$ & $15 \%$ & $13 \%$ & $51 \%$ & $100 \%$ \\
\hline Media & $4,959.80$ & $47,163.00$ & $67,551.00$ & $9,060.00$ & $91,618.40$ & $81,634.40$ & $313,046.00$ & \\
\hline
\end{tabular}

Fuente: elaboración propia con base en datos de la Subdelegación de Pesca, BCS. 
En 2013, en la oficina de Santa En el comparativo se puede obserRosalía se reportó un total de var que, en la evolución del perío$\$ 2.051 .225$ (tabla 21). Un $87 \%$ se do 2009-2013, la oficina de Santa obtuvo entre los meses de marzo y abril y de octubre a diciembre.

A todo lo identificado mediante el uso de la estadística descriptiva, debe agregarse que la producción de ostión por oficina se calculó de igual forma en los últimos cinco años. Los resultados de este análisis se indican en la tabla 22 y en la figura 18 . Rosalía generó la mitad de la producción del estado de BCS, con una media aproximada de 313.046 toneladas anuales de ostión. Por otra parte, la oficina de Ciudad Constitución reportó solo un $11 \%$ de la producción de los últimos cinco años, con una media aproximada de 67.551 toneladas anuales de ostión.

Figura 18. Análisis comparativo de la producción histórica generada en el período 2009-2013, en las oficinas del estado de BCS

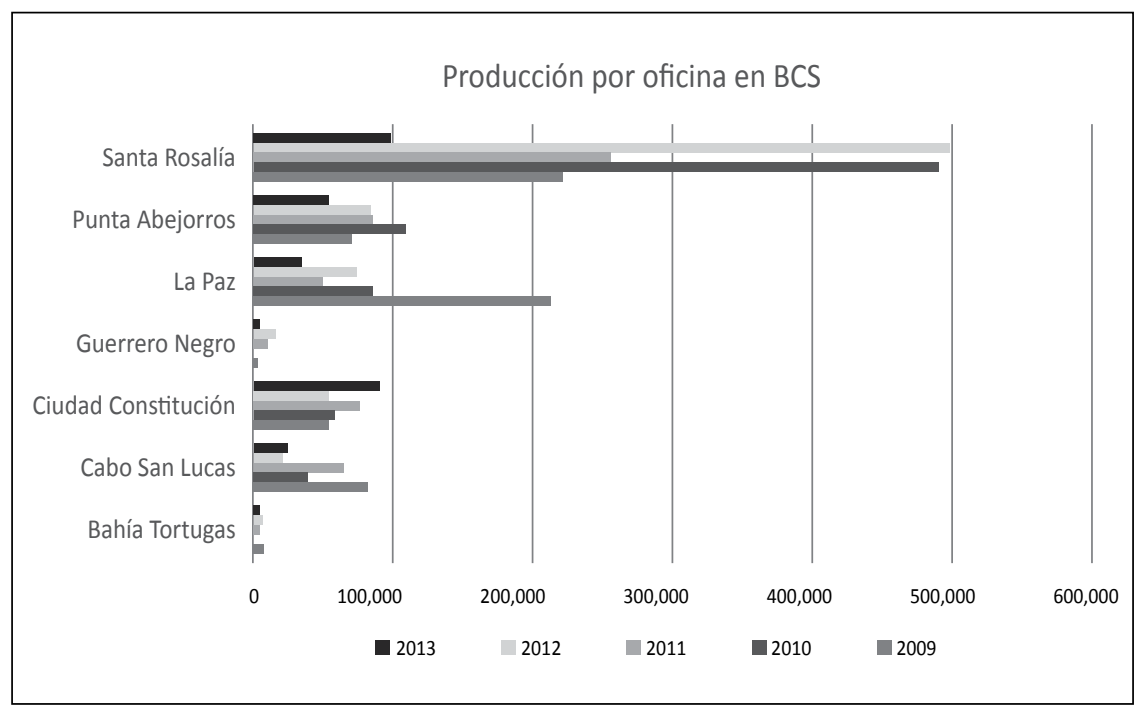

Fuente: elaboración propia con base en datos de la Subdelegación de Pesca, BCS.

\section{CONCLUSIONES}

El desarrollo del presente trabajo generó como principales aportes, el co- nocimiento de la producción de ostión japonés en el estado de BCS, con el fin de apoyar la generación de una planeación estratégica adecuada, 
conforme a los recursos naturales y organizacionales con que se cuenta en los municipios. Desde una perspectiva natural, es posible empezar a planear los programas de comercialización que deberán aplicarse por parte de la iniciativa privada, así como de los organismos públicos inmersos en el tema de la acuicultura en el estado.

En el segmento público, existe un organismo descentralizado de la Sagarpa y de la Conapesca, denominado Sistema Producto Ostión de Baja California Sur. Este organismo puede desarrollar mecanismos de control (indicadores) para medir con claridad la producción que podría ser comercializada en el escenario local y asesorar a los productores sobre los requisitos que deberían cumplir en caso de trasladar su producto al extranjero.

Además, debe mencionarse que, si a partir de estos datos se puede visualizar un esquema empresarial de exportación, es necesario que el productor local refuerce los mecanismos tecnológicos a su disposición, así como sus recursos humanos, logísticos y financieros y que se acerque a instituciones de apoyo, de forma que su experiencia comercial sea fructífera.

\section{REFERENCIAS}

Bourne, N. (1979). Pacific oyster crassostrea gigas in british columbia and the south pacific islands. En R. Mann (ed.), Exotic species in mariculture. Proceedings of a symposium on exotic species in mariculture: Case histories of the japanese oyster, crassostrea gigas (thunberg), with implications for other fisheries (pp. 1-53). Cambridge: MIT Press.

Chew, K. (1979). The pacific oyster crassostrea gigas in the west coast of the united states. En R. Mann (Ed.), Exotic species in mariculture. Proceedings of a symposium on exotic species in mariculture: Case histories of the japanese oyster, crassostrea gigas (thunberg), with implications for other fisheries (pp. 5482). Cambridge: MIT Press.

Hernández Sampieri, R., Fernández Collado, C. \& Baptista Lucio, P. (2004). Metodología de la investigación (3a ed.). México D. F.: McGraw-Hill.

Islas Olivares, R. (1975). El ostión japonés Crassostrea gigas en Baja California. Revista Ciencias Marinas, 2(1), 58-99.

Islas Olivares, R. (1982). Análisis económico en el cultivo del os- 
tión japonés (Crassostrea gigas) en Puerto Don Juan, Bahía de Los Ángeles, B. C. Ciencias Marinas, 8(2), 55-68.

Mann, R. (ed.) (1979). Exotic species in mariculture. Proceedings of a symposium on exotic species in mariculture: Case his- tories of the japanese oyster, crassostrea gigas (thunberg), with implications for other fisheries (pp. 1-53). Cambridge: MIT Press.

Stickney, R. R. (1979). Principles of warmwater aquaculture. Nueva York-Chichester: Wiley. 